\title{
Measurement of the magnetophoretic velocity of different superparamagnetic beads
}

\author{
Stefan Achtsnicht ${ }^{1}$, Kristina Schönenborn ${ }^{1}$, Andreas Offenhäusser ${ }^{1}$, and Hans-Joachim Krause ${ }^{1, *}$ \\ ${ }^{1}$ Institute of Complex Systems, Bioelectronics (ICS-8), Forschungszentrum Jülich, Germany \\ *e-mail: h.-j.krause@fz-juelich.de
}

\begin{abstract}
The movement of magnetic beads due to a magnetic field gradient is of great interest in different application fields. In this report we present a technique based on a magnetic tweezers setup to measure the velocity factor of magnetically actuated individual superparamagnetic beads in a fluidic environment. Several beads can be tracked simultaneously in order to gain and improve statistics. Furthermore we show our results for different beads with hydrodynamic diameters between 200 and $1000 \mathrm{~nm}$ from diverse manufacturers. These measurement data can, for example, be used to determine design parameters for a magnetic separation system, like maximum flow rate and minimum separation time, or to select suitable beads for fixed experimental requirements.
\end{abstract}

Keywords: magnetophoretic velocity, superparamagnetic bead, magnetic tweezers, magnetic separation, magnetic actuation

\section{Introduction}

In modern bioanalytical and biomedical applications, magnetic beads are widely used [1] [2]. They can be detected with a variety of different sensors, based for example on giant magnetoresistance (GMR), atomic magnetometers (AM), superconducting quantum interference devices (SQUIDS) and techniques like frequency mixing and nuclear magnetic resonance (NMR). In these techniques, the beads usually act as labels. They can also be used as handles [3], for example to clean a solution [4] [5] or to deliver substances to a specific target [6]. In [7], it was shown that they can be used to enhance the adeno-associated viral vector delivery in human neural stem cell infection. Furthermore, they are used in sample preparation and isolation techniques [8] [9] [10], like immunomagnetic separation (IMS) [11]. Their use as handles and labels can also be combined. In such an application, they can first be used to enhance the sample concentration and then to measure it. With this combination, also smaller concentrations can be detected, and a bigger volume can be scanned instead of a smaller subsample.

Therefore the magnetophoretic velocity of different magnetic beads is of great interest in different application fields. Other groups have, for example, measured the magnetophoretic velocity of magnetic particles by observing the leading edge of the collection of particles placed inside a tube filled with glycerol [12] or by measuring the turbidity of a suspension containing particles [13] [14]. It has been shown that cooperative effects can happen, which lead to a much bigger velocity of particles in highly concentrated samples than in lower ones [15] [16] [17]. Because of this, it is very important to know the bead's characteristics at a concentration which fits the later application. A three-dimensional vector model describing how three individual beads interact with each other in a static fluid in a uniform magnetic field is presented in [18]. In [19], the motion of magnetic beads in excised tissue due to a magnetic field was investigated by placing the tissue above a magnet and the magnetic beads on top of it. Afterwards the tissue has been sliced to measure the distance they have moved within a given time. In [20] a microscope consisting of two poles which is generated by two pairs of permanent magnets has been used to measure the magnetophoretic mobility of different cells bound to one type of a paramagnetic carrier. Other works analysed magnetic glass microspheres with diameters in the range of 2-11 $\mu \mathrm{m}[21]$ and magnetic beads $(1-5 \mu \mathrm{m})$ [22].

In this report, we present a technique and measurement results of the determination of the magnetophoretic velocity of different commercially available magnetic beads in a fluidic environment. 
Because we observe the velocity of individual beads, we are able to show the deviations within one bead type as well. Furthermore, we can make sure that we just analyse individual beads and not clustered ones. Because of this, we can use this technique to measure the velocity in low concentration situations, for instance during the removal of pathogens from a drinking water sample and subsequent detection of the pathogen amount with the help of magnetic beads.

\section{Method}

To measure the magnetic mobility of different magnetic beads from different suppliers, we have developed and implemented a methodology, which is described in the following.

\section{Theory}

A magnetic moment $\vec{m}$ in a magnetic field $\vec{B}$ experiences a magnetic force $\vec{F}_{\text {mag }}$

$$
\vec{F}_{\text {mag }}=(\vec{m} \cdot \nabla) \cdot \vec{B} \text {. }
$$

The beads in this study are superparamagnetic. As long as the magnetic field is small enough that the beads are still in their linear regime of their magnetization curve, the induced magnetic moment $\vec{m}$ is proportional to the magnetic field $\vec{B}$ and can be expressed as

$$
\vec{m}=\frac{V_{b} \chi}{\mu_{0}} \vec{B} .
$$

In our experiment, the beads are not always in the linear magnetization region, but always below saturation. $V_{b}$ is the volume of the bead, $\chi$ its effective magnetic susceptibility and $\mu_{0}$ the vacuum permeability.

By using these two formulas, the magnetic force can be expressed as [10]

$$
\vec{F}_{\text {mag }}=\frac{V_{b} \chi}{2 \mu_{0}} \nabla|\vec{B}|^{2} .
$$

It can be seen that the force on a bead is proportional to the gradient of the squared absolute value of the magnetic field.

As the bead is moving in a fluidic environment, its movement will lead to a frictional drag force. In the case of spherical beads under laminar conditions, this force can be expressed with the Stokes law

$$
\vec{F}_{\text {drag }}=6 \pi \eta R_{\text {hydr }} \vec{v} . \quad \text { Eq. } 4
$$

Here, $\vec{v}$ denotes the bead's velocity, $\eta$ the viscosity of the surrounding liquid, and $R_{h y d r}$ the bead's hydrodynamic radius.

In a system where only $\vec{F}_{\text {mag }}$ and $\vec{F}_{\text {drag }}$ are affecting a bead's vertical movement, the resultant velocity of the bead due to the magnetic field gradient can be expressed as

$$
\vec{v}=\frac{V_{b} \chi}{12 \pi \mu_{0} \eta R_{h y d r}} \nabla|\vec{B}|^{2} .
$$

The proportionality constant between $\nabla|\vec{B}|^{2}$ and $\vec{v}$ in Eq. 5 is the so called bead factor $f_{b}$ divided by the viscosity $\eta$ :

$$
f_{b}=\frac{V_{b} \chi}{12 \pi \mu_{0} R_{h y d r}} .
$$

Thus, the velocity of a bead becomes simply

$$
\vec{v}=\frac{f_{b}}{\eta} \nabla|\vec{B}|^{2}
$$


For our measurements, we additionally defined a velocity factor which denotes the change of the velocity $\Delta v$ of a bead due to a changing current $\Delta /$ in our magnetic tweezers setup.

$$
f_{v}=\frac{\Delta v}{\Delta I}
$$

Beads in a liquid environment will also experience Brownian motion. Their lateral displacement in two dimensions can be described as follows [18]

$$
\left\langle\Delta r^{2}(t)\right\rangle_{2 D}=\frac{2 k_{B} T}{3 R_{h y d r} \pi \eta} t .
$$

Here, $k_{B}$ is the Boltzmann constant, $T$ the absolute temperature in Kelvin, and $t$ the time scale of observation. This formula shows that the displacement due to Brownian motion scales with the inverse square root of the viscosity, therefore it will get smaller when the viscosity of the surrounding media becomes larger.

A higher viscosity will therefore lead to a lower velocity of the beads, which facilitates optical tracking of the beads' trajectories because they can be traced in a higher number of frames, and their displacement due to Brownian motion will become smaller. Also, with a higher viscosity, the sedimentation of the beads due to gravity will be reduced.

\section{Setup}

For measuring the bead movement, we decided to use a magnetic tweezers setup. We modified the setup which is described in detail in [23] to be used with only 2 tips. Because we need a magnetic field gradient in one straight direction, two tips are sufficient. We can easily change the magnetic gradient by applying different currents over the coils. Both coils have 750 windings and a resistance of $5.5 \Omega$. In principle, it possible to generate a magnetic field gradient with just one tip connected to an electromagnet, but then the field lines will focus towards the tip edge in a star-shaped manner. Therefore, we use two tips where the second one is supplied with a current which is half as big as the other's. With this technique, we generate a magnetic gradient which exhibits quasi-parallel magnetic field lines in the center area between the tips. Because both tips have a different current, we move the point where no gradient is acting on the bead away from the centre between both tips. The coils are placed on a magnetic yoke, which directs the magnetic field towards the sample holder. The sample solution is placed inside a fluidic cell with two tips which do not directly contact the yoke. On top of a glass cover slip, the 2 magnetic tips made of VACOFLUX ${ }^{\otimes} 50$ foil (Vacuumschmelze GmbH \& Co. KG, Germany) are glued with a distance of about $2 \mathrm{~mm}$. On top of these, a glass ring is fixed to hold the liquid during the measurement. This cell is fixed with a 3D-printed holder on top of the yoke. The whole setup is shown in Fig. 1. 


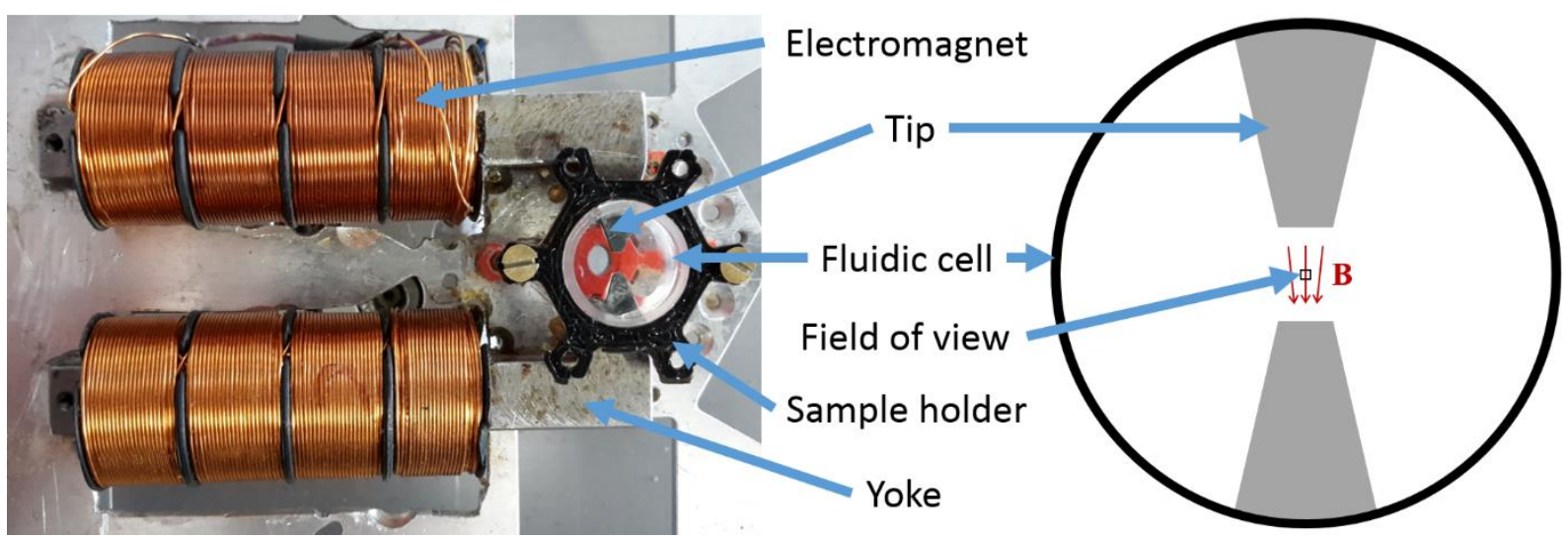

Fig. 1: Left: Picture of the setup to measure the velocity factor of different magnetic beads by microscopic optical image tracking. The setup consists of two electromagnets fixed on a yoke, which directs the magnetic field towards the fluidic cell. In the fluidic cell, two tips are fixed which genereate a magnetic field gradient with quasi-parallel field lines in the middle between them. Right: Sketch of the fluidic cell with its tips, the field of view during measurements with the microscope and magnetic field lines (red).

The sample is illuminated from the bottom with a custom-built light source via an optical fiber. Because the viscosity depends on the temperature, we took care to limit the heat contribution from our light emitting diode (LED) to a tolerable value. The light-source consists of a 3 Watt cool white LED (SparkFun Electronics, Inc, COM-13105) which is connected to constant current source (up to $330 \mathrm{~mA}$, SparkFun Electronics, Inc, COM-13716). This source is powered with a $12 \mathrm{~V}$ power supply. A small program on the PC and the microcontroller (Pololu A-Star 32U4 Micro) allows the operator to adjust the light intensity. The LED is fixed on top of a heatsink and has a custom-made casing to direct the light emitted by the light source to the optical fibre.

With the help of this magnetic tweezers setup and optical image processing of the magnetic tweezers microscope videos, the trajectories of individual beads at different magnetic gradients were tracked. The bead speeds are determined, the velocity factor (Eq. 8) and the bead factor (see Eq. 6), a bead property independent of fluid viscosity and field gradient, is calculated. Care is taken that only individual beads are tracked in order to exclude cooperative effects like chain formation. From the tracking of multiple individual beads, statistics of one bead type is obtained within one measurement run. The measurements were performed with an optical resolution of $0.198 \mu \mathrm{m} /$ pixel.

\section{Sample preparation}

The study was performed with magnetic beads produced by different manufacturers. The beads had a hydrodynamic diameter in the size range of 200 to $1000 \mathrm{~nm}$. An overview of the measured beads is given in Table 1. Due to their size, all of these beads can be optically observed with a light microscope.

The preparation of the sample solution was always done according to the following recipe. First the beads inside their original sample containers where vortexed to get a homogeneous bead concentration in the sample. Then $30 \mu \mathrm{l}$ of this bead solution was added to $3 \mathrm{ml}$ Diethylene glycol (DEG, Diethylene glycol BioUltra, Sigma-Aldrich). Afterwards, the suspension was mixed, again with a vortexer. It needs to be taken into account that because of the higher viscosity of DEG, the vortexing time needed to get a homogeneous concentration is longer than in an aqueous solution. The concentration was chosen such that it is high enough to detect multiple beads during one measurement run, and at the same time low enough so that the beads are not affected too much by the field deformations resulting from other beads inside the sample and that the chance of forming clusters of beads is reduced. At a temperature of $298.15 \mathrm{~K}$, DEG has a viscosity of $26.812 \mathrm{mPa} \cdot \mathrm{s}$ while water has only $0.981 \mathrm{mPa} \cdot \mathrm{s}$ according to [24]. Therefore, the measured velocity and the lateral 
displacement due to Brownian motion will be about a factor of 27 smaller than in water, according to Eq. 5 and Eq. 9.

\section{Measurement and data processing}

About $200 \mu$ of the bead suspension was pipetted into the fluidic cell. Videos of the bead movements were recorded at 6 different currents over the coil which correspond to different magnetic gradients. For each bead type, the same field gradients were used. After the measurements, the recorded videos were processed. Just beads which fulfil the following conditions are selected for tracking. The beads need to move due to the magnetic field gradient, they should be freely moving, should not cluster with other beads, and should be visible in several video frames to get a proper tracking with sufficient frame statistics.

With the position of each bead in each frame and the time stamps of each frame, we calculated the frame-to-frame velocities of each bead and determined its mean velocity. Because we have many beads at the same time, we can also calculate the mean velocity of all tracked beads and their standard deviation. An example plot of the velocity as a function of the current for bead type "Mono Mag Streptavidin", produced by Ocean NanoTech LLC. with a hydrodynamic diameter of $1000 \mathrm{~nm}$, is shown in Fig. 2. Additionally a linear fit, which considers the standard deviation, is added. We can see that in this case, the measurement points can be described quite well with a linear function $\left(R^{2}: 0.98\right)$ and a slope of $237.6 \pm 13.8 \mu \mathrm{m} /$ As. This slope is now called the velocity factor.

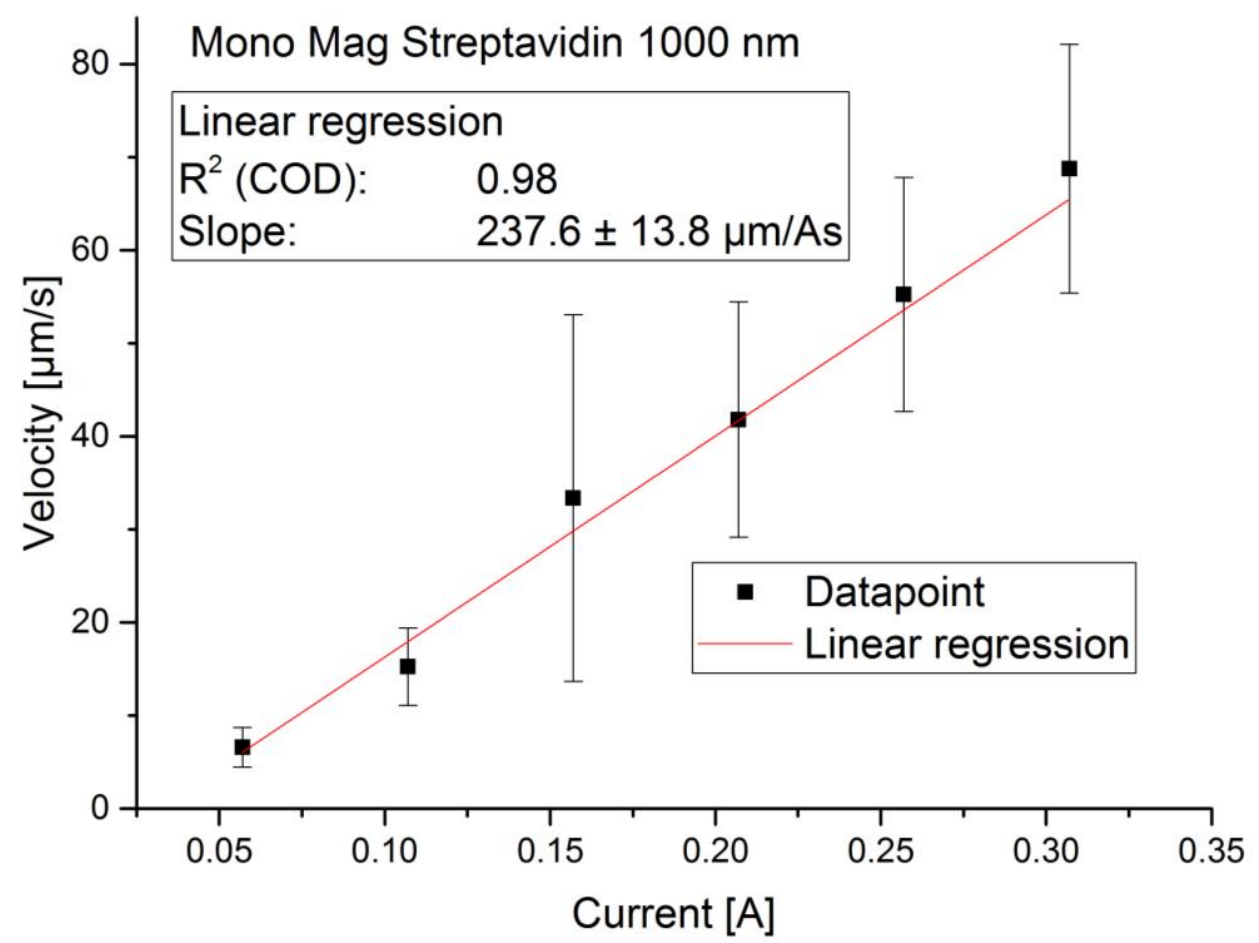

Fig. 2. Velocity of the bead Mono Mag Streptavidin $1000 \mathrm{~nm}$ in respect to the current at the electromagnet. A linear fit is added to determine the bead's velocity factor (slope).

Following this method, the velocity factors of different beads from the following companies have been determined within this research: Chemical $\mathrm{GmbH}$, micromod Partikeltechnologie $\mathrm{GmbH}$, microParticles $\mathrm{GmbH}$, and Ocean NanoTech, LLC. All these beads are listed together with their hydrodynamic diameter and their determined velocity factor together with its standard deviation in Table 1. The hydrodynamic diameter was taken from the datasheet provided by the manufacturers. 
Table 1. Overview of the measured beads. Listed are the bead type, manufacturer and the hydrodynamic diameter, as stated by the manufacturer. The determined velocity factor, its standard deviation and the $R$ squared is given.

\begin{tabular}{cccccc}
\hline \hline Bead name & Manufacturer & $\begin{array}{c}\text { Hydrodynamic } \\
\text { diameter }[\mathrm{nm}]\end{array}$ & $\begin{array}{c}\text { Velocity factor } \\
{[\mu \mathrm{m} / \mathrm{As}]}\end{array}$ & $\begin{array}{c}\text { Standard deviation } \\
{[\mu \mathrm{m} / \mathrm{As}]}\end{array}$ & $\begin{array}{c}\mathrm{R}^{2} \\
(\mathrm{COD})\end{array}$ \\
\hline \hline FluidMAG-SA $200 \mathrm{~nm}$ & Chemicell & 200 & 305.4 & 23.8 & 0.98 \\
\hline ScreenMag SA $500 \mathrm{~nm}$ & Chemicell & 500 & 232.4 & 13.9 & 0.99 \\
\hline SiMAG-SA $1000 \mathrm{~nm}$ & Chemicell & 1000 & 158.9 & 22.1 & 0.93 \\
\hline beadMAG $1000 \mathrm{~nm}$ & Chemicell & 1000 & 174.8 & 20.2 & 0.95 \\
\hline nanomag-CLD SA $200 \mathrm{~nm}$ & Micromod & 300 & 60.7 & 16.9 & 0.93 \\
\hline nanomag-CLD SA $500 \mathrm{~nm}$ & Micromod & 500 & 260.3 & 26.7 & 0.96 \\
\hline nanomag-D SA $500 \mathrm{~nm}$ & Micromod & 500 & 266.5 & 91.3 & 0.68 \\
\hline PS-MAG-SA-S1978 & microParticles & 360 & 126.9 & 16.7 & 0.94 \\
\hline PS-MAG-SA-S1979-1 & microParticles & 536 & 385.1 & 51.8 & 0.93 \\
\hline Mono Mag SA 500 nm & Ocean NanoTech & 500 & 58.7 & 33.3 & 0.44 \\
\hline Mono Mag SA $1000 \mathrm{~nm}$ & Ocean NanoTech & 1000 & 237.6 & 13.8 & 0.98 \\
\hline Hi-Sur SA 1000 nm & Ocean NanoTech & 1000 & 269.5 & 28.8 & 0.96 \\
\hline \hline
\end{tabular}

To estimate the magnetic field gradient in our setup, we also measured the magnetic beads Dynabeads M-280 and M-450 from Thermo Fisher Scientific. Their velocity factors were found as 22.9 and $111.8 \mu \mathrm{m} /$ As, respectively. Additional information about the beads which was needed for our analysis was taken from [25] and is listed in Table 2.

Table 2. Properties of the magnetic beads Dynabeads M-280 and M-450 from Thermo Fisher Scientific according to $[25]$.

\begin{tabular}{lcc}
\hline \hline Parameter & $\begin{array}{c}\text { Dynabeads M-280 (Thermo Fisher } \\
\text { Scientific) }\end{array}$ & $\begin{array}{c}\text { Dynabeads M-450 (Thermo Fisher } \\
\text { Scientific) }\end{array}$ \\
\hline \hline Hydrodynamic radius $R_{\text {hydr }}[\mu \mathrm{m}]$ & 1.4 & 2.2 \\
\hline Density $\rho\left[\mathrm{g} / \mathrm{cm}^{3}\right]$ & 1.4 & 1.6 \\
\hline Mass susceptibility $\chi_{m}\left[\mathrm{~m}^{3} / \mathrm{kg}\right]$ & $54 \times 10^{-5}$ & $102 \times 10^{-5}$ \\
\hline \hline
\end{tabular}

Here, the mass susceptibility $\chi_{m}$ of the beads is given. The effective susceptibility of the whole bead is given as

$$
\chi=\rho \chi_{m} . \quad \text { Eq. } 10
$$

Taking the total (hydrodynamic) volume of a spherical bead, $V_{h y d r}=\frac{4}{3} \pi R_{h y d r}{ }^{3}$, the bead factor (Eq. 6) becomes

$$
f_{b}=\frac{\chi R_{h y d r^{2}}}{9 \mu_{0}} .
$$

For Dynabeads M-280 beads with $R_{h y d r}=1.4 \mu \mathrm{m}$ and an effective susceptibility of $\chi=\rho \chi_{m}=0.756$, the bead factor becomes $f_{b}=1.3 \cdot 10^{-7} \frac{A m}{T}$.

For M-280, an average velocity of $4.59 \mu \mathrm{m} / \mathrm{s}$ was found when applying a current of $0.2 \mathrm{~A}$. 
Using the identity $\vec{v}=\frac{f_{b}}{\eta} \nabla|\vec{B}|^{2}$ and calculating the factor of M-280 beads in DEG (viscosity $\left.\eta=26.8 \cdot 10^{-3} \mathrm{~Pa} \cdot \mathrm{s}\right)$ to $\frac{f_{b}}{\eta}=4.9 \cdot 10^{-6} \frac{A m}{P a s T}$, it is concluded that the magnetic field gradient in the magnetic tweezers setup is about $\nabla|\vec{B}|^{2}=1 \frac{T^{2}}{m}$ at $0.2 \mathrm{~A}$ current.

To verify this, we performed the same calculations with the velocity of M-450 beads of $22.36 \mu \mathrm{m} / \mathrm{s}$ at $0.2 \mathrm{~A}$ and also got a magnetic field gradient of about $\nabla|\vec{B}|^{2}=1 \frac{T^{2}}{m}$.

Using this magnetic field gradient, we can also calculate the bead factor (which is a characteristics of the bead type, independent of the experimental setup) for each bead as

$$
f_{b}=\eta \frac{I}{\nabla|\vec{B}|^{2}} f_{v}
$$

with $\frac{I}{\nabla|\vec{B}|^{2}}=\frac{0.2 \mathrm{~A}}{1 \frac{T^{2}}{m}}=0.2 \frac{\mathrm{Am}}{T^{2}}$ for our setup.

Using the results of this work, the velocity of a specific bead at a given magnetic field gradient and a viscous medium can be calculated by using Eq. 7. In Fig. 3, this velocity is shown for all measured beads as a function of their hydrodynamic size, as given by the manufacturer. For the calculation of the velocities of different beads, the specifications of our setup (beads in DEG and a current of $0.2 \mathrm{~A}$, which results in a magnetic field gradient of about $1 \mathrm{~T} / \mathrm{m}^{2}$ ) where used. In addition, the bead factor is given for each bead.

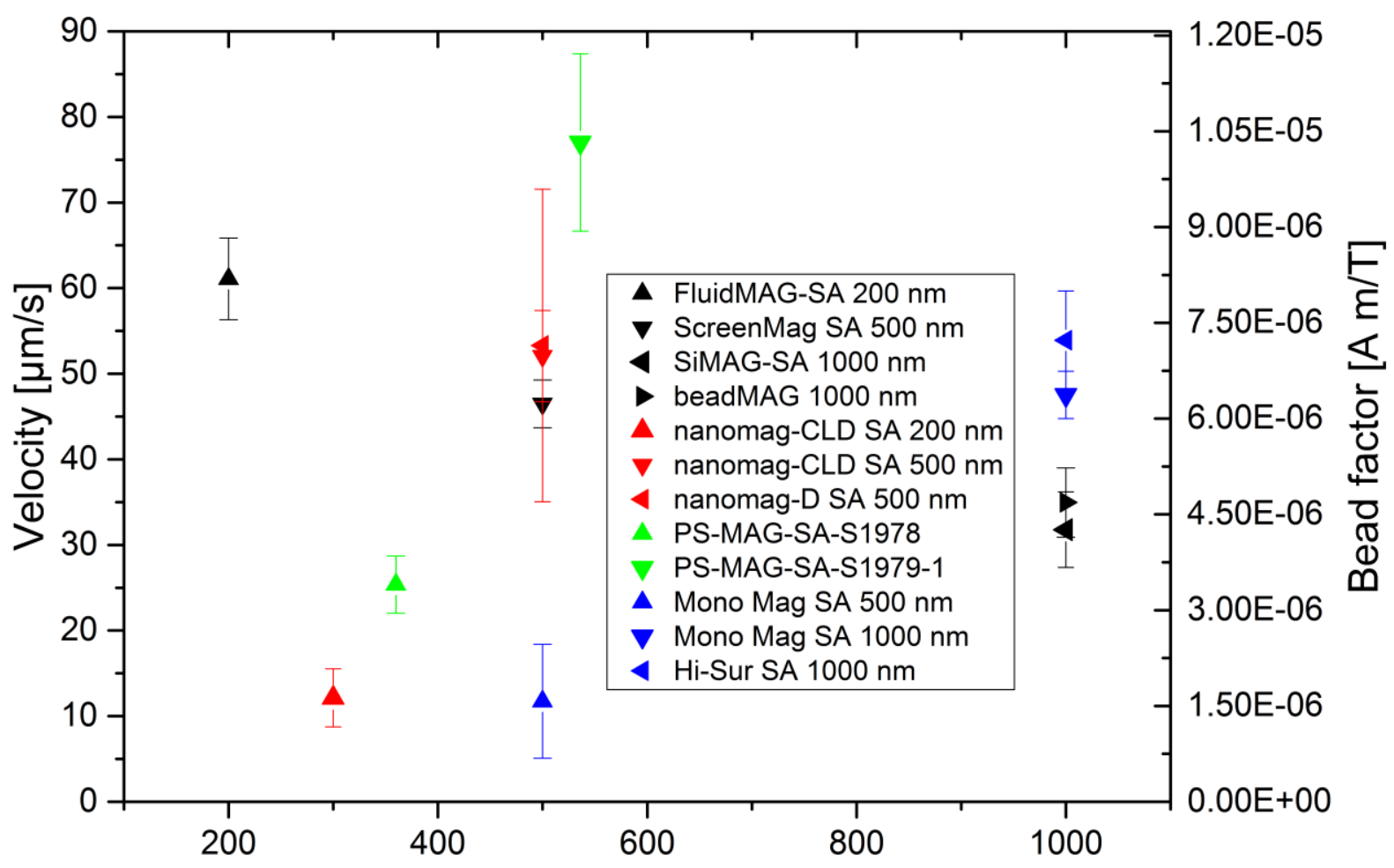

Hydrodynamic diameter [nm] (manufacturer's data)

Fig. 3. Plot of velocity and bead factor, depending on the hydrodynamic diameter of the bead as provided by the manufacturer. The velocities are calculated for beads moving in DEG and a magnetic field gradient of $1 \mathrm{~T}^{2} / \mathrm{m}$. The different manufacturers are color-coded while the different bead types from one manufacturer are marked with different symbols. For each bead type, also the standard deviation of the velocity factors are given. Manufacturers: Chemicell (black), micromod (red), microParticles (green), and Ocean NanoTech (blue). 
It can be seen that there is a factor of about 6.6 between the slowest and the fastest bead. At a hydrodynamic diameter of $500 \mathrm{~nm}$, the measured values for the two beads from micromod which differ in their shell (dextran and cross-linked dextran) and their Magnetite content (75-80\% vs $80-90 \%$ ) have quite similar velocity factors, but their standard deviation varies much more. If you compare their velocities with the one from Ocean NanoTech "Mono Mag SA $500 \mathrm{~nm}$ ", there is a factor of about 4.5 among them. While all the beads of Chemicell show a decreasing velocity with increasing hydrodynamic diameter, all the beads from the companies micromod Partikeltechnologie $\mathrm{GmbH}$, microParticles $\mathrm{GmbH}$ and Ocean NanoTech, LLC. exhibit the inverse behaviour. With an increase in bead size, also their velocities increase. This behaviour is consistent with previously reported results for other magnetic beads like for magnetic glass microspheres with diameters in the range of 2-11 $\mu \mathrm{m}$ [21] or for other beads (1-5 $\mu \mathrm{m})$ [22].

These measurement data can, for example, be used to determine design parameters for a magnetic separation system, like maximum flow rate and minimum separation time, or to select suitable beads for fixed experimental requirements.

\section{Acknowledgements}

Discussions with Christof Steiner and Jennifer Laun (DITABIS AG, Pforzheim, Germany) and with Florian Schröper (Fraunhofer IME, Aachen, Germany) are gratefully acknowledged.

\section{Funding}

Part of this research was supported by the Federal Ministry of Education and Research (BMBF) [grant number 13N13712]. The funding source had no involvement in study design; in the collection, analysis and interpretation of data; in the writing of the report; and in the decision to submit the article for publication.

\section{Competing interest statement}

The authors have no competing interest to declare.

\section{References}

[1] Y.-T. Chen, A. G. Kolhatkar, O. Zenasni, S. Xu and T. R. Lee, "Biosensing Using Magnetic Particle Detection Techniques," Sensors, vol. 17, p. 2300, 2017.

[2] Q. A. Pankhurst, J. Connolly, S. K. Jones and J. Dobson, "Applications of magnetic nanoparticles in biomedicine," Journal of Physics D: Applied Physics, vol. 36, p. R167, 2003.

[3] X. Hu, R. Abedini-Nassab, B. Lim, Y. Yang, M. Howdyshell, R. Sooryakumar, B. B. Yellen and C. Kim, "Dynamic trajectory analysis of superparamagnetic beads driven by on-chip micromagnets," Journal of Applied Physics, vol. 118, p. 203904, 2015.

[4] M. FRANZREB, "Magnettechnologie in der Verfahrenstechnik wässriger Medien," Wissenschaftliche Berichte FZKA, vol. 6916, pp. A--212, 2003.

[5] M. Möller, A. Hermann, R. Groß, M.-O. Diesner, P. Küppers, W. Luther, N. Malanowski, D. Haus and A. Zweck, Nanomaterialien: Auswirkungen auf Umwelt und Gesundheit, 2013, pp. 35,141. 
[6] C. Alexiou and R. Jurgons, "Magnetic Drug Targeting," in Magnetism in Medicine, Wiley-VCH Verlag GmbH \& Co. KGaA, 2007, pp. 596-605.

[7] E. Kim, J.-S. Oh, I.-S. Ahn, K. I. Park and J.-H. Jang, "Magnetically enhanced adeno-associated viral vector delivery for human neural stem cell infection," Biomaterials, vol. 32, pp. 8654-8662, 2011.

[8] I. Šafařík and M. Šafaříková, "Use of magnetic techniques for the isolation of cells," Journal of Chromatography B: Biomedical Sciences and Applications, vol. 722, pp. 33-53, 1999.

[9] M. Apel, U. A. O. Heinlein, S. Miltenyi, J. Schmitz and J. D. M. Campbell, "Magnetic Cell Separation for Research and Clinical Applications," in Magnetism in Medicine, Wiley-VCH Verlag GmbH \& Co. KGaA, 2007, pp. 571-595.

[10] M. Zborowski, "Physics of Magnetic Cell Sorting," in Scientific and Clinical Applications of Magnetic Carriers, U. Häfeli, W. Schütt, J. Teller and M. Zborowski, Eds., Boston, MA: Springer US, 1997, pp. 205-231.

[11] J. Chen and B. Park, "Effect of immunomagnetic bead size on recovery of foodborne pathogenic bacteria," International Journal of Food Microbiology, vol. 267, pp. 1-8, 2018.

[12] V. S. Kalambur, B. Han, B. E. Hammer, T. W. Shield and J. C. Bischof, "In vitro characterization of movement, heating and visualization of magnetic nanoparticles for biomedical applications," Nanotechnology, vol. 16, p. 1221, 2005.

[13] K. Witte, K. Müller, C. Grüttner, F. Westphal and C. Johansson, "Particle size- and concentration-dependent separation of magnetic nanoparticles," Journal of Magnetism and Magnetic Materials, vol. 427, pp. 320-324, 2017.

[14] F. Yu, L. Zhang, Y. Huang, K. Sun, A. E. David and V. C. Yang, "The magnetophoretic mobility and superparamagnetism of core-shell iron oxide nanoparticles with dual targeting and imaging functionality," Biomaterials, vol. 31, pp. 5842-5848, 2010.

[15] J. Lim, S. P. Yeap, C. H. Leow, P. Y. Toh and S. C. Low, "Magnetophoresis of iron oxide nanoparticles at low field gradient: The role of shape anisotropy," Journal of Colloid and Interface Science, vol. 421, pp. 170-177, 2014.

[16] J. Faraudo and J. Camacho, "Cooperative magnetophoresis of superparamagnetic colloids: theoretical aspects," Colloid and Polymer Science, vol. 288, pp. 207-215, 0112010.

[17] J. S. Andreu, J. Camacho, J. Faraudo, M. Benelmekki, C. Rebollo and L. M. Martinez, "Simple analytical model for the magnetophoretic separation of superparamagnetic dispersions in a uniform magnetic gradient," Phys. Rev. E, vol. 84, no. 2, p. 021402, 82011.

[18] O. Oduwole and S. Sheard, "Three-dimensional mathematical modelling of magnetic bead interactions within a magnetic separation system," Applied Mathematical Modelling, vol. 45, pp. 123-133, 2017.

[19] S. Kulkarni, B. Ramaswamy, E. Horton, S. Gangapuram, A. Nacev, D. Depireux, M. Shimoji and B. Shapiro, "Quantifying the motion of magnetic particles in excised tissue: Effect of particle 
properties and applied magnetic field," Journal of Magnetism and Magnetic Materials, vol. 393, pp. 243-252, 2015.

[20] J. J. Chalmers, Y. Zhao, M. Nakamura, K. Melnik, L. Lasky, L. Moore and M. Zborowski, "An instrument to determine the magnetophoretic mobility of labeled, biological cells and paramagnetic particles," Journal of Magnetism and Magnetic Materials, vol. 194, pp. 231-241, 1999.

[21] U. O. Häfeli, R. Ciocan and J. P. Dailey, "Characterization of magnetic particles and microspheres and their magnetophoretic mobility using a digital microscopy method," European Cells and Materials, vol. 3. Suppl. 2, pp. 24-27, 2002.

[22] C. Zhou, E. D. Boland, P. W. Todd and T. R. Hanley, "Magnetic particle characterizationmagnetophoretic mobility and particle size," Cytometry Part A, vol. 89, pp. 585-593, 2016.

[23] L. Chen, A. Offenhäusser and H.-J. Krause, "Magnetic tweezers with high permeability electromagnets for fast actuation of magnetic beads," Review of Scientific Instruments, vol. 86, p. 044701, 2015.

[24] T. M. Aminabhavi and B. Gopalakrishna, "Density, Viscosity, Refractive Index, and Speed of Sound in Aqueous Mixtures of N,N-Dimethylformamide, Dimethyl Sulfoxide, N,NDimethylacetamide, Acetonitrile, Ethylene Glycol, Diethylene Glycol, 1,4-Dioxane, Tetrahydrofuran, 2-Methoxyethanol, and 2-Ethoxyethanol at $298.15 \mathrm{~K}$," Journal of Chemical \& Engineering Data, vol. 40, pp. 856-861, 1995.

[25] G. Fonnum, C. Johansson, A. Molteberg, S. Mørup and E. Aksnes, "Characterisation of Dynabeads ${ }^{\circledR}$ by magnetization measurements and Mössbauer spectroscopy," Journal of Magnetism and Magnetic Materials, vol. 293, pp. 41-47, 2005. 